\title{
WEAK CONVERGENCE OF HIGH-SPEED NETWORK TRAFFIC MODELS
}

\author{
SIDNEY RESNICK AND ERIC VAN DEN BERG
}

\begin{abstract}
We consider a network traffic model consisting of an infinite number of sources linked to a server. Sources initiate transmissions to the server at Poisson time points. The duration of each transmission has a heavy tailed distribution. We show that suitable scalings of the traffic process converge to a totally skewed stable Lévy motion in Skorohod space, equipped with the Skorohod $M_{1}$ topology. This allows us to prove a heavy traffic theorem for a single server fluid model.
\end{abstract}

\section{INTRODUCTION}

In modern telecommunications traffic data, features such as long range dependence, self-similarity, and heavy tails are prominent. Furthermore, the characteristics of the data vary greatly over measurements at different sites and different points in time. This is especially true for internet data, as described in e.g. [32]. Black box time series modeling, which has been succesful in finite variance (low variability) settings, is inadequate for modeling teletraffic datasets. See e.g. $[25,20,24,23,9,21,6,7]$ and the discussions therein. Hence, we should attempt more structural modeling. The size of recent data sets of traffic network measurements, the dynamic nature of communication networks, and the complexity of the traffic they carry argue strongly in favor of parsimonious and structural network traffic models.

As a more structural traffic model, a superposition of a large number of ON/OFF type sources whose activity periods are heavy tailed, has received considerable attention. Such models are approximated by fluid models with $\mathrm{M} / \mathrm{G} / \infty$ inputs, sometimes referred to as infinite source Poisson models. (Cf. $[13,14,16,15,18,33,34,35,28,19,5]$.) The $\mathrm{M} / \mathrm{G} / \infty$ input model described in [17] is of this type. Using a distributional limit theorem, Konstantopoulos and Lin ([17]) explain the suitability of an totally skewed stable Lévy motion as a macroscopic traffic model for a high-speed network switch. The limit process is self-similar, but somewhat surprisingly has independent increments. The marginal distribution of the Lévy stable motion matches those sometimes empirically obtained in practice ([11]). Also, results on the queue length process of queueing systems with Lévy inputs are reported in [17].

However, in [17] the convergence of the scaled traffic model to the limit Lévy motion is only shown to occur in the sense of finite dimensional distributions. To validate application of the limit model in telecom applications, it is important to establish convergence in a stronger sense of functional weak convergence in a suitable topology. Typically, when the limit process has almost surely continuous paths, one uses the Skorohod $J_{1}$ topology, or even the uniform topology. Konstantopoulos and Lin discuss why scaled infinite source Poisson models cannot converge in $\left(D[0, \infty), J_{1}\right)$ to a Lévy limit. A comprehensive discussion of these issues is in the recently released reports of Whitt [30, 31, 29].

The main result of the present paper shows that indeed, the scaled and centered cumulative traffic process converges to a skewed stable Lévy motion in $D[0, \infty)$ equipped with the Skorohod

This research was partly supported by NSF Grant DMS-97-04982 at Cornell University. S. Resnick was also partially supported by NSA grant MDA904-98-1-0041 at Cornell. Portions of this work were completed at the University of North Carolina, Chapel Hill, and the hospitality of the Department of Statistics is gratefully acknowledged. 
$M_{1}$ topology. This result is then applied to obtain a heavy traffic limit theorem for a single server fluid queue.

The paper is organized as follows. In Section 2, the network traffic model considered is described in detail, and basic definitions and notation are given. Our assumptions, weaker than those used in [17], are explained. In Section 3, we give an alternative proof of finite dimensional convergence based on point process methods. This paves the way for proving weak $M_{1}$ convergence in $D[0, \infty)$, which is done in Section 4, using ideas from [2,3]. Finally, in Section 5, the convergence results are applied to obtain a heavy traffic theorem for a single server fluid model with the given input traffic.

\section{Infinite source Poisson model}

We consider the $\mathrm{M} / \mathrm{G} / \infty$ input model of incoming traffic to a communication network. Let $\left\{\Gamma_{k}, k \geq 1\right\}$ denote the points of a homogeneous Poisson process on $[0, \infty)$ with rate $\lambda$. Suppose at time $\Gamma_{k}$, a source starts a transmission, and continues to transmit for a period of length $L_{k}$, at possibly time-varying rate. Let $\zeta(t), t \in[0, \infty)$ be a monotone increasing, right continuous function describing the transmission schedule; the total amount of data transmitted by source $k$ in time $t-\Gamma_{k}$ is given by $\zeta\left(\left(t-\Gamma_{k}\right) \wedge L_{k}\right)$. Assume for convenience that $\zeta(s)=0$ for $s<0$. The total volume of traffic injected into the network between 0 and $t$ is

$$
A(t)=\sum_{k=1}^{\infty} \zeta\left(\left(t-\Gamma_{k}\right) \wedge L_{k}\right), t \geq 0 .
$$

We make the following assumptions on the distribution of $L_{k}$, and the schedule function $\zeta$ : Suppose

$$
P\left[L_{k}>x\right]=1-F(x)=x^{-\alpha} L(x), 1<\alpha<2, x>0
$$

for some slowly varying function $L(x)$, i.e. $L(t x) / L(t) \rightarrow 1$ as $t \rightarrow \infty$. Further, assume $\zeta$ is regularly varying with index $\beta$, that is:

$$
\lim _{t \rightarrow \infty} \frac{\zeta(x t)}{\zeta(t)}=x^{\beta}, \frac{\alpha}{2}<\beta<\alpha .
$$

These assumptions subsume the case where sources transmit at unit rate; that is, $\zeta(t)=t$ and $\beta=1$. Define

$$
b(t):=\inf \left\{x: P\left[\zeta\left(L_{1}\right)>x\right] \leq t^{-1}\right\} .
$$

It is easy to see, that $\zeta\left(L_{k}\right)$ has regularly varying tail probabilities with index $-\alpha / \beta$ since $P\left[\zeta\left(L_{1}\right)>\right.$ $x] \sim 1-F\left(\zeta^{\leftarrow}(x)\right)$ is a composition of two regularly varying functions and is hence regularly varying $([8,12,4,26])$. It follows that $b(t)$ is regularly varying with index $\beta / \alpha$.

Since $\alpha>1$ and $\alpha / \beta>1$,

$$
\mu:=E\left(L_{1}\right)=\int_{0}^{\infty} x F(d x)<\infty, \quad \mu_{\zeta}:=E\left(\zeta\left(L_{1}\right)\right)=\int_{0}^{\infty} \zeta(x) F(d x)<\infty .
$$

\section{Finite dimensional Convergence: A point process approach}

We now give a version of [17, Theorem 4] without some extraneous assumptions assumed in [17]. Our proof of this result, based on a point process approach, will help to show convergence in $D[0, \infty)$ endowed with Skorohod's $M_{1}$ topology. 
Theorem 3.1. Suppose $A(t)$ is defined by (2.1), b(t) by (2.4) and $\mu_{\zeta}$ by (2.5). Assume (2.2) and (2.3) hold. Then

$$
\frac{A(T t)-\lambda T t \mu_{\zeta}}{b(T)} \stackrel{f . d . d .}{\longrightarrow} X_{\alpha / \beta}(t) \text { as } T \rightarrow \infty
$$

where $X_{\alpha / \beta}(t)$ is $\alpha / \beta$ stable Lévy motion with mean 0, skewness parameter 1 , and scaling parameter $\left(\lambda C_{\alpha / \beta}^{-1}\right)^{\beta / \alpha}$, where $C_{\alpha / \beta}$ is defined in (3.18). Here $\stackrel{\text { f.d.d. }}{\longrightarrow}$ denotes convergence of the finitedimensional distributions.

Proof. As a first step, we will show:

$$
\frac{A(T)-\lambda T \mu_{\zeta}}{b(T)} \Rightarrow X_{\alpha / \beta}(1)
$$

The point process

$$
M=\sum_{k=1}^{\infty} \epsilon_{\left(\Gamma_{k}, L_{k}\right)}=\operatorname{PRM}(\lambda d t \times F(d x)),
$$

is a Poisson process on $[0, \infty) \times[0, \infty]$ with mean measure $\lambda d t \times F(d x)([26])$. Consider the cumulative input in $[0, T]$, denoted by $A(T)$. We can divide this input in two parts: the traffic from sources that have started and stopped transmitting before time $T$, and the traffic from sources that have started transmitting before $T$, and are still transmitting at $T$. Define (see Figure 1)

$$
R_{1}^{(T)}=\{(x, y) \in[0, \infty) \times[0, \infty]: x+y \leq T\}, \quad R_{2}^{(T)}=\{(x, y) \in[0, \infty) \times[0, \infty]: x \leq T \leq x+y\} .
$$

Then

$$
\begin{aligned}
A(T) & =\sum_{k=1}^{\infty} \zeta\left(L_{k}\right) 1_{\left[\left(\Gamma_{k}, L_{k}\right) \in R_{1}^{(T)}\right]}+\sum_{k=1}^{\infty} \zeta\left(T-\Gamma_{k}\right) 1_{\left[\left(\Gamma_{k}, L_{k}\right) \in R_{2}^{(T)}\right]} \\
& =A_{1}(T)+A_{2}(T) .
\end{aligned}
$$

Since $A_{1}(T)$ and $A_{2}(T)$ are functions of $M$ over the disjoint regions $R_{1}^{(T)}$ and $R_{2}^{(T)}, A_{1}(T)$ and $A_{2}(T)$ are independent.

Observe that,

$$
E\left(M\left(R_{1}^{(T)}\right)\right)=\int_{s=0}^{T} \lambda d s \int_{y=0}^{T-s} F(d y)=\lambda \hat{F}(T) \rightarrow \infty,
$$

where $\hat{F}(T):=\int_{0}^{T} F(s) d s \sim T F(T) \sim T$, as $T \rightarrow \infty$, and

$$
E\left(M\left(R_{2}^{(T)}\right)\right)=\int_{0}^{T} \lambda d s \int_{y=T-s}^{\infty} F(d y)=\lambda \int_{0}^{T} \bar{F}(T-s) d s=\lambda m(T) \stackrel{T \rightarrow \infty}{\longrightarrow} \lambda \mu,
$$

with $m(T):=\int_{0}^{T} \bar{F}(s) d s$. Also $M$ restricted to $R_{1}^{(T)}$ is almost surely finite and hence has representation

$$
\left.M\right|_{R_{1}^{(T)}} \stackrel{d}{=} \sum_{k=1}^{P_{1}(T)} \epsilon_{\left(\tau_{k}^{(T)}, J_{k}^{(T)}\right)}
$$




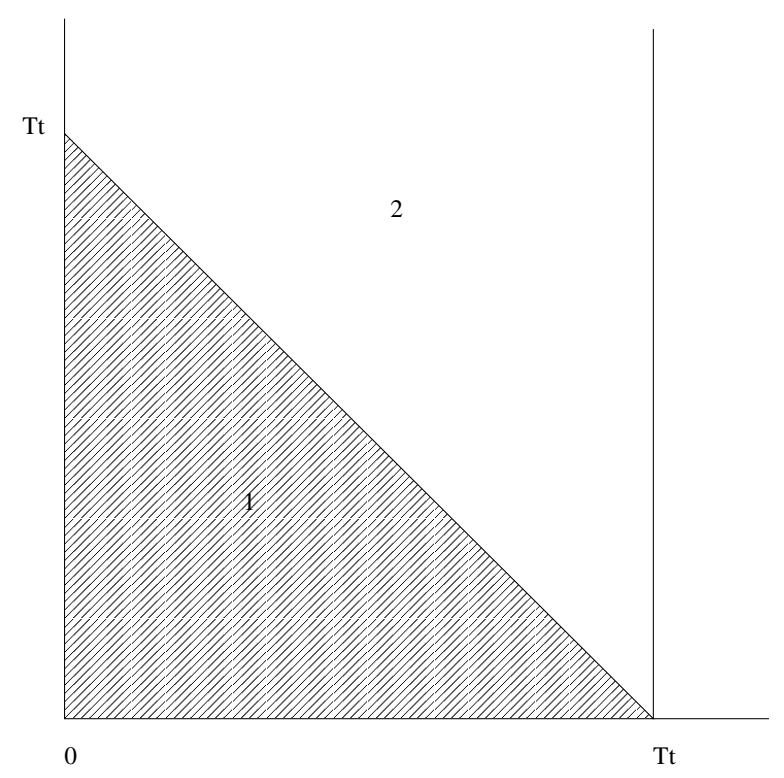

Figure 1. Traffic up to time $T$ divided in two parts

where $P_{1}(T)$ is Poisson with parameter $\lambda \hat{F}(T)$, independent of the i.i.d. random pairs $\left\{\left(\tau_{k}^{(T)}, J_{k}^{(T)}\right)\right\}$. The joint distribution of $\left(\tau_{k}^{(T)}, J_{k}^{(T)}\right)$ is the normalized mean measure

$$
\left.\frac{\lambda d s F(d y)}{\lambda \hat{F}(T)}\right|_{R_{1}^{(T)}}=\frac{d s F(d y)}{\hat{F}(T)}
$$

and therefore the distribution of $J_{k}^{(T)}$ satisfies for $x<T$

$$
P\left[J_{k}^{(T)} \leq x\right]=\frac{\int_{0}^{T-x} d s F(x)+\int_{T-x}^{T} d s F(T-s)}{\hat{F}(T)}=\frac{(T-x) F(x)+\hat{F}(x)}{\hat{F}(T)}
$$

and for $x<T$

$$
P\left[J_{k}^{(T)}>x\right]=\frac{(T-x) \bar{F}(x)-\int_{x}^{T} \bar{F}(s) d s}{\hat{F}(T)} .
$$

We can write

$$
A_{1}(T) \stackrel{d}{=} \sum_{k=1}^{P_{1}(T)} \zeta\left(J_{k}^{(T)}\right)
$$

Next, we will analyze the tail behavior of $\zeta\left(J_{k}^{(T)}\right)$ as $T \rightarrow \infty$. Note that $0 \leq J_{k}^{(T)} \leq T$, and hence $\zeta(0) \leq \zeta\left(J_{k}^{(T)}\right) \leq \zeta(T)$. We have

$$
\begin{aligned}
P\left(\zeta\left(J_{k}^{(T)}\right)>b(T) x\right) & =P\left(J_{k}^{(T)}>\zeta^{\leftarrow}(b(T) x)\right) \\
& =\frac{\left\{\left(T-\zeta^{\leftarrow}(b(T) x)\right) \bar{F}\left(\zeta^{\leftarrow}(b(T) x)\right)-\int_{\zeta^{\leftarrow}(b(T) x)}^{T} \bar{F}(s) d s\right\}}{\hat{F}(T)} .
\end{aligned}
$$


Now $\hat{F}(T) \sim T$ as $T \rightarrow \infty$. Since $\zeta^{\leftarrow}$ is regularly varying with index $1 / \beta$, and $b(t)$ is regularly varying with index $\beta / \alpha$, we have

$$
\zeta^{\leftarrow}(b(T) x) \sim x^{1 / \beta} \zeta^{\leftarrow}(b(T)) \sim x^{1 / \beta} T^{1 / \alpha} L^{*}(T)
$$

for a slowly varying function $L^{*}$, and recalling $1<\alpha<2$ we see that

$$
\frac{T}{\zeta^{\leftarrow}(b(T) x)} \rightarrow \infty, \quad T \rightarrow \infty .
$$

Putting things together, we get

$$
\begin{aligned}
T P\left(\zeta\left(J_{k}^{(T)}>b(T) x\right)\right. & \sim\left(1-\frac{\zeta^{\leftarrow}(b(T) x)}{T}\right) T \bar{F}\left(\zeta^{\leftarrow}(b(T) x)\right)-\int_{\zeta^{\leftarrow}(b(T) x)}^{T} \bar{F}(s) d s \\
& \sim(1+o(1)) x^{-\alpha / \beta}-\frac{\zeta^{\leftarrow}(b(T) x)}{T} \int_{1}^{T / \zeta^{\leftarrow}(b(T) x)} T \bar{F}\left(\zeta^{\leftarrow}(b(T) x) s\right) d s \\
& \rightarrow x^{-\alpha / \beta}-0 \int_{1}^{\infty} x^{-\alpha / \beta} s^{-\alpha} d s=x^{-\alpha / \beta} .
\end{aligned}
$$

This result is equivalent to (see [26])

$$
\operatorname{TP}\left(\frac{\zeta\left(J_{k}^{(T)}\right.}{b(T)} \in \cdot\right) \stackrel{v}{\rightarrow} \eta(\cdot)
$$

where $\eta$ is the Lévy measure given by $\eta(d x)=\alpha / \beta x^{-\alpha / \beta-1} 1_{(0, \infty]}(x)$. Using Karamata's theorem, we can verify that

$$
\limsup _{T \rightarrow \infty} T \operatorname{Var}\left(\frac{\zeta\left(J_{1}^{(T)}\right)}{b(T)} 1_{\left(\left|\frac{\zeta\left(J_{1}^{(T)}\right)}{b(T)}\right| \leq M\right)}\right)<\infty
$$

for any $M>0$,

$$
\lim _{\epsilon \downarrow 0} \limsup _{T \rightarrow \infty} T \operatorname{Var}\left(\frac{\zeta\left(J_{1}^{(T)}\right)}{b(T)} 1_{\left(\left|\frac{\zeta\left(J_{1}^{(T)}\right)}{b(T)}\right| \leq \epsilon\right)}\right)<\infty
$$

and

$$
\lim _{M \rightarrow \infty} \limsup _{T \rightarrow \infty} T E\left(\left|\frac{\zeta\left(J_{1}^{(T)}\right)}{b(T)}\right| 1_{\left(\left|\frac{\zeta\left(J_{1}^{(T)}\right)}{b(T)}\right|>M\right)}\right)=0
$$

Therefore, as in Section 2 of [22], it follows that in $\left(D[0, \infty), J_{1}\right)$,

$$
S_{T}(t):=\left(\sum_{k=1}^{[T t]} \frac{\zeta\left(J_{k}^{(T)}\right)}{b(T)}-[T t] E\left(\frac{\zeta\left(J_{k}^{(T)}\right)}{b(T)} 1_{\left(\zeta\left(J_{k}^{(T)}\right) \leq b(T)\right)}\right) \Rightarrow S_{\alpha / \beta}(t)\right.
$$

where $S_{\alpha / \beta}(t)$ is skewed $\alpha / \beta$ stable Lévy motion. $S_{\alpha / \beta}(1)$ is a stable random variable with skewness parameter 1 and scaling parameter $C_{\alpha / \beta}^{-\beta / \alpha}$, where

$$
C_{\alpha / \beta}=\frac{1-\frac{\alpha}{\beta}}{\Gamma\left(2-\frac{\alpha}{\beta}\right) \cos \left(\frac{\pi \alpha}{2 \beta}\right)} .
$$


Furthermore, from the central limit theorem for Poisson random variables,

$$
B_{T}:=\frac{\left(P_{1}(T)-\lambda \hat{F}(T)\right)}{\sqrt{\lambda \hat{F}(T)}} \Rightarrow B(1)
$$

where $B(1)$ has a $\mathcal{N}(0,1)$ distribution, and so $P_{1}(T) / T \stackrel{P}{\rightarrow} \lambda$. Consequently from (3.17) and (3.19)

$$
\begin{aligned}
\frac{A_{1}(T)}{b(T)} & \stackrel{d}{=} \sum_{k=1}^{T P_{1}(T) / T} \frac{\zeta\left(J_{k}^{(T)}\right)}{b(T)}=S_{T}\left(\frac{P_{1}(T)}{T}\right)+P_{1}(T) E\left(\frac{\zeta\left(J_{k}^{(T)}\right)}{b(T)} 1_{\left(\zeta\left(J_{k}^{(T)}\right) \leq b(T)\right)}\right) \\
& =\left(S_{\alpha / \beta}(\lambda)+o_{p}(1)\right)+\frac{\sqrt{\lambda \hat{F}(T)} B_{T}+\lambda \hat{F}(T)}{b(T)} E\left(\zeta\left(J_{k}^{(T)}\right) 1_{\left(\zeta\left(J_{k}^{(T)}\right) \leq b(T)\right)},\right.
\end{aligned}
$$

so

$$
\frac{A_{1}(T)}{b(T)}-\frac{\lambda \hat{F}(T)}{b(T)} E\left(\zeta\left(J_{k}^{(T)}\right) 1_{\left(\zeta\left(J_{k}^{(T)}\right) \leq b(T)\right)}=S_{\alpha / \beta}(\lambda)+\frac{\sqrt{\lambda \hat{F}(T)}}{b(T)} B_{T}+o_{p}(1) \Rightarrow S_{\alpha / \beta}(\lambda)\right.
$$

as $T \rightarrow \infty$. Note that $b(T) \sim T^{\beta / \alpha} L_{2}(T)$, and $\beta>\alpha / 2$, so that $\sqrt{\hat{F}(T)} / b(T) \sim T^{1 / 2} / b(T) \rightarrow 0$.

Also, observe that $S_{\alpha / \beta}(\lambda) \stackrel{d}{=} \lambda^{\beta / \alpha} S_{\alpha / \beta}(1)$, so $S_{\alpha / \beta}(\lambda)$ is a stable random variable with scaling parameter $\left(\lambda C_{\alpha / \beta}^{-1}\right)^{\beta / \alpha}$. Next, we show:

Proposition 3.1. With $A(t)$ defined as in (2.1), b(t) as in (2.4), and $A_{1}(t)$ as in (3.3), we have

$$
\frac{A(T)-A_{1}(T)}{b(T)} \stackrel{P}{\rightarrow} 0
$$

as $T \rightarrow \infty$.

Proof. By (3.3) we need to show:

$$
\frac{A_{2}(T)}{b(T)} \stackrel{P}{\rightarrow} 0
$$

We can write:

$$
\begin{gathered}
A_{2}(T) \stackrel{d}{=} \sum_{k=1}^{P_{2}(T)} \zeta\left(T-\tau_{k}^{(T)}\right), \\
\left.M\right|_{R_{2}^{(T)}} \stackrel{d}{=} \sum_{k=1}^{P_{2}(T)} \epsilon_{\left(\tau_{k}^{(T)}, \eta_{k}^{(T)}\right)}
\end{gathered}
$$

where $P_{2}(T)$ is Poisson with parameter $\lambda m(T) \stackrel{T \rightarrow \infty}{\longrightarrow} \lambda \mu$, and $P_{2}(T)$ is independent of the points $\left\{\left(\tau_{k}, \eta_{k}\right), k \geq 1\right\}$, which are in turn i.i.d. with distribution

$$
\left.\frac{\lambda d s F(d y)}{\lambda m(T)}\right|_{R_{2}(T)}=\left.\frac{d s F(d y)}{m(T)}\right|_{R_{2}(T)} .
$$

For $x<T$,

$$
P\left(T-\tau_{1}^{(T)} \leq x\right)=P\left(\tau_{1}^{(T)} \geq T-x\right)=\frac{\int_{s=T-x}^{T} \lambda d s \bar{F}(T-s)}{\lambda m(T)}=\frac{m(x)}{m(T)}
$$


It suffices to show $E A_{2}(T) / b(T) \rightarrow 0$. We have

$$
\begin{aligned}
E A_{2}(T) / b(T) & =\lambda m(T) E \zeta\left(T-\tau_{1}^{(T)}\right) / b(T) \\
& \sim \lambda \int_{0}^{T} \zeta(u) \bar{F}(u) d u / b(T) \\
& \sim(\text { const }) \zeta(T) T \bar{F}(T) / b(T) .
\end{aligned}
$$

We are done if we show

$$
\beta+1-\alpha<\beta / \alpha
$$

or equivalently if $\beta \alpha+\alpha-\alpha^{2}<\beta$. However

$$
\beta \alpha-\alpha(\alpha-1)<\beta \alpha-\beta(\alpha-1)=\beta,
$$

which proves the statement.

Combining Proposition 3.1 and (3.20), we have

$$
\frac{A(T)}{b(T)}-\lambda \frac{\hat{F}(T)}{b(T)} E\left(\zeta\left(J_{k}^{(T)}\right) 1_{\left[\zeta\left(J_{k}^{(T)}\right) \leq b(T)\right]}\right) \Rightarrow S_{\alpha / \beta}(\lambda)
$$

as $T \rightarrow \infty$.

In order to center $A(T)$ by $\lambda T \mu_{\zeta}$ we first subtract

$$
\begin{aligned}
\lambda \frac{\hat{F}(T)}{b(T)} \mu_{\zeta}-\lambda \frac{\hat{F}(T)}{b(T)} E\left(\zeta\left(J_{k}^{(T)}\right) 1_{\left[\zeta\left(J_{k}^{(T)}\right) \leq b(T)\right]}\right) & =\lambda \hat{F}(T) E\left(\frac{\zeta\left(J_{k}^{(T)}\right)}{b(T)} 1_{\left[\zeta\left(J_{k}^{(T)}\right)>b(T)\right]}\right. \\
& +\lambda \frac{\hat{F}(T)}{b(T)}\left(\mu_{\zeta}-\mu_{\zeta}^{(T)}\right),
\end{aligned}
$$

where $\mu_{\zeta}^{(T)}=E\left(\zeta\left(J_{1}^{(T)}\right)\right.$. Now

$$
\begin{aligned}
\lambda \frac{\hat{F}(T)}{b(T)}\left(\mu_{\zeta}-\mu_{\zeta}^{(T)}\right) & =\lambda \frac{\hat{F}(T)}{b(T)}\left[\iint_{\substack{0 \leq s \leq T \\
\hat{x}<\infty}} \zeta(x) \frac{d s F(d x)}{T}-\iint_{\substack{0 \leq s \leq T \\
x \leq T-s}} \zeta(x) \frac{d s F(d x)}{\hat{F}(T)}\right] \\
& =\lambda \frac{\hat{F}(T)}{b(T)}\left[\iint_{\substack{0 \leq s \leq T \\
x<\infty}} \zeta(x) \frac{d s F(d x)}{\hat{F}(T)} \cdot\left(1+\frac{\hat{F}(T)}{T}-1\right)-\iint_{\substack{0 \leq s \leq T \\
x \leq T-s}} \zeta(x) \frac{d s F(d x)}{\hat{F}(T)}\right] \\
& =\lambda \frac{\hat{F}(T)}{b(T)}\left[\iint_{\substack{0 \leq s \leq T \\
x>T-s}} \zeta(x) \frac{d s F(d x)}{\hat{F}(T)}\right]+\frac{\lambda}{b(T)} T \mu_{\zeta}\left(\frac{\hat{F}(T)}{T}-1\right) \\
& =\frac{\lambda}{b(T)} \iint_{\substack{0 \leq s \leq T \\
x>T-s}} \zeta(x) d s F(d x)+\frac{\lambda}{b(T)} \mu_{\zeta}(\hat{F}(T)-T) \\
& =I+I I .
\end{aligned}
$$

We have

$$
|I I|=\frac{\lambda}{(T)} \mu_{\zeta} \int_{0}^{T} \bar{F}(u) d u \leq \frac{\lambda \mu_{\zeta} \mu}{b(T)} \rightarrow 0
$$


and

$$
\begin{aligned}
I= & \frac{\lambda}{b(T)} \int_{s=0}^{T}\left(\int_{x=T-s}^{\infty} \zeta(x) F(d x)\right) d s \\
& \sim(\text { const }) \frac{\zeta(T) \bar{F}(T) T}{b(T)} \rightarrow 0
\end{aligned}
$$

by regular variation of $\zeta(\cdot)$ and $b(\cdot)$, and (3.24). To summarize, for large $T$,

$$
\mu_{\zeta}-\mu_{\zeta}^{(T)} \leq(\text { const }) \zeta(T) \bar{F}(T)+2 \lambda \mu \mu_{\zeta} / T
$$

We may conclude

$$
\lambda \frac{\hat{F}(T)}{b(T)} \mu_{\zeta}-\lambda \frac{\hat{F}(T)}{b(T)} E\left(\zeta\left(J_{k}^{(T)}\right) 1_{\left[\zeta\left(J_{k}^{(T)}\right) \leq b(T)\right]}\right)=\lambda \hat{F}(T) E\left(\frac{\zeta\left(J_{k}^{(T)}\right)}{b(T)} 1_{\left[\zeta\left(J_{k}^{(T)}\right)>b(T)\right]}+o(1) .\right.
$$

Since $\hat{F}(T) \sim T$ and $T P\left(\zeta\left(J_{k}^{(T)}\right)>b(T)\right) \rightarrow 1$, we have from the Lemma on page 578 of [10], a variant of Karamata's theorem, that

$$
\lambda \hat{F}(T) E\left(\frac{\zeta\left(J_{k}^{(T)}\right)}{b(T)} 1_{\left[\zeta\left(J_{k}^{(T)}\right)>b(T)\right]}\right) \rightarrow \lambda\left(\frac{\frac{\alpha}{\beta}}{\frac{\alpha}{\beta}-1}\right) .
$$

Furthermore,

$$
\begin{aligned}
\frac{\hat{F}(T) \mu_{\zeta}-\lambda T \mu_{\zeta} \lambda}{b(T)} & =\frac{\left(\int_{0}^{T} F(s) d s-T\right) \mu_{\zeta} \lambda}{b(T)} \\
& =\frac{-\lambda \mu_{\zeta} \int_{0}^{T} \bar{F}(s) d s}{b(T)} \sim \frac{-\lambda \mu_{\zeta} \mu}{b(T)} \\
& \rightarrow 0,
\end{aligned}
$$

as $T \rightarrow \infty$. We conclude:

$$
\frac{A(T)-\lambda T \mu_{\zeta}}{b(T)} \Rightarrow S_{\alpha / \beta}(\lambda)-\lambda\left(\frac{\alpha / \beta}{\alpha / \beta-1}\right)=: X_{\alpha / \beta}(1),
$$

where $X_{\alpha / \beta}(1)$ is an $\alpha / \beta$ stable random variable with mean 0 , skewness 1 , and scaling parameter $\left(\lambda C_{\alpha / \beta}^{-1}\right)^{-\beta / \alpha}$.

Now, consider $A(T t), t>0$ fixed. Recalling that $b(t)$ is regularly varying with index $\beta / \alpha$, it follows that:

$$
\frac{A(T t)-\lambda T t \mu_{\zeta}}{b(T)}=\frac{b(T t)}{b(T)} \frac{A(T t)-\lambda T t \mu_{\zeta}}{b(T t)} \Rightarrow t^{\beta / \alpha} X_{\alpha / \beta}(1) \stackrel{d}{=} X_{\alpha / \beta}(t)
$$

as $T \rightarrow \infty$.

Next, we show joint convergence. We content ourselves with considering two time points $0 \leq$ $s<t$. We divide the traffic between 0 and $T t$ into parts as illustrated in Figure 2.

From Proposition 3.1 we have

$$
\left(\frac{A(T s)-\lambda \mu_{\zeta} T s}{b(T)}, \frac{A(T t)-\lambda \mu_{\zeta} T t}{b(T)}\right)=\left(\frac{A_{1}(T s)-\lambda T s}{b(T)}, \frac{A_{1}(T t)-\lambda T t}{b(T)}\right)+\left(o_{p}(1), o_{p}(1)\right) .
$$




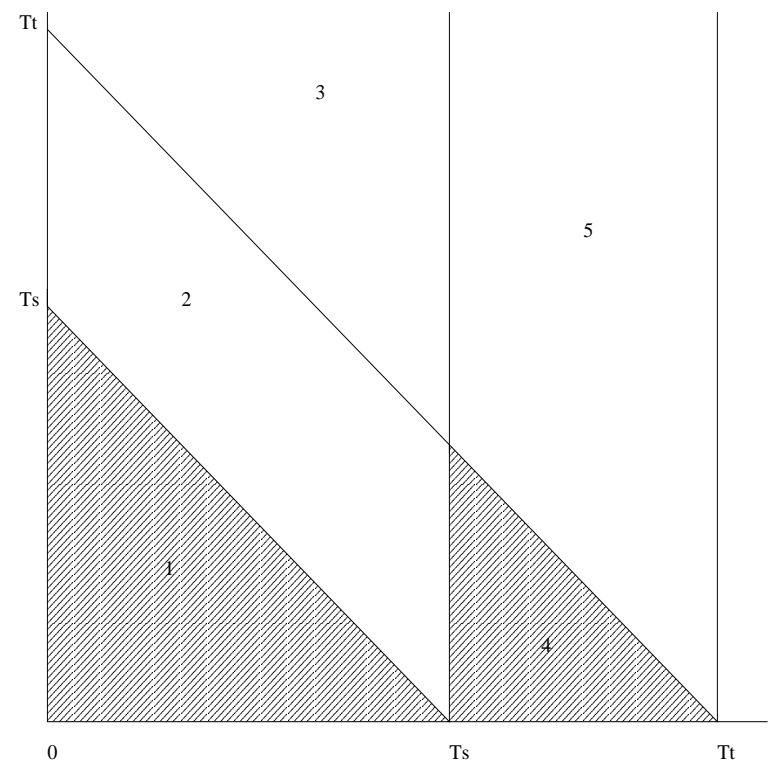

Figure 2. Traffic up to time $T t$ divided in five parts

Now

$$
\begin{aligned}
A_{1}(T t) & =A_{1}(T s)+\iint_{\substack{T s<u \leq T t \\
0 \leq u+y \leq T t}} \zeta(y) M(d u, d y)+\iint_{\substack{0<u \leq T s \\
T s \leq u+y \leq T t}} \zeta(y) M(d u, d y) \\
& =: A_{1}(T s)+A_{4}^{\prime}+A_{2}^{\prime},
\end{aligned}
$$

where the three summands are independent. Observe that by scaling

$$
A_{4}^{\prime} \stackrel{d}{=} A_{1}(T(t-s))
$$

and $A_{2}^{\prime}$ is negligible since

$$
\begin{aligned}
\frac{E\left(A_{2}^{\prime}\right)}{b(T)} & =\frac{1}{b(T)} E\left(\iint_{\substack{0<u \leq T s \\
T s \leq u+y \leq T t}} \zeta(y) M(d u, d y)\right) \\
& =\frac{1}{b(T)} \iint_{\substack{0<u \leq T s \\
T s \leq u+y \leq T t}} \zeta(y) \lambda d u F(d y) \\
& =\frac{\lambda}{b(T)} \int_{u=0}^{T s} \int_{y=T s-u}^{T t-u} \zeta(y) d u F(d y) \\
& =\frac{\lambda T \zeta(T) \bar{F}(T)}{b(T)} \int_{u=0}^{s} \int_{y=s-u}^{t-u} \frac{\zeta(T y)}{\zeta(T)} \frac{F(T d y)}{\bar{F}(T)} d u \\
& =c_{T}(s, t) \frac{T \zeta(T) \bar{F}(T)}{b(T)} \\
& \sim c(s, t) \frac{T \zeta(T) \bar{F}(T)}{b(T)},
\end{aligned}
$$


where

$$
c(s, t)=\frac{\alpha}{(\alpha-\beta)(\beta-\alpha+1)}\left[s^{\beta-\alpha+1}-t^{\beta-\alpha+1}+(t-s)^{\beta-\alpha+1}\right] .
$$

This ratio goes to 0 due to (3.24) as required. The joint convergence then follows from (3.30), (3.31), (3.32).

Remark 3.1. For later purposes, note from the definitions of $c_{T}(s, t)$ and $c(s, t)$ that for $s<t$, these functions are increasing in $t$ and decreasing in $s$.

Note that the tail behavior of the random variables $J_{k}^{(T t)}$ as $T \rightarrow \infty$ does not depend on the value of $t$. To check this, note that $0 \leq J_{k}^{(T t)} \leq T t$, so $\zeta(0) \leq \zeta\left(J_{k}^{(T)}\right) \leq \zeta(T t)$, and from (3.8),

$$
\begin{aligned}
P\left(\zeta\left(J_{k}^{(T t)}\right)>b(T) x\right) & =P\left(J_{k}^{(T t)}>\zeta^{\leftarrow}(b(T) x)\right) \\
& =\frac{\left\{\left(T t-\zeta^{\leftarrow}(b(T) x)\right) \bar{F}\left(\zeta^{\leftarrow}(b(T) x)\right)-\int_{\zeta \leftarrow(b(T) x)}^{T t} \bar{F}(s) d s\right\}}{\hat{F}(T t)} .
\end{aligned}
$$

As $T \rightarrow \infty, \hat{F}(T t) \sim T t$. Now

$$
\begin{aligned}
T P\left(\zeta\left(J_{k}^{(T t)}>b(T) x\right)\right. & \sim\left(1-\frac{\zeta^{\leftarrow}(b(T) x)}{T t}\right) T \bar{F}\left(\zeta^{\leftarrow}(b(T) x)\right)-\frac{1}{t} \int_{\zeta^{\leftarrow}(b(T) x)}^{T t} \bar{F}(s) d s \\
& \sim(1+o(1)) x^{-\alpha / \beta}-\frac{\zeta^{\leftarrow}(b(T) x)}{T t} \int_{1}^{T t / \zeta^{\leftarrow(b(T) x)}} T \bar{F}\left(\zeta^{\leftarrow}(B(T) x) s\right) d s \\
& \rightarrow x^{-\alpha / \beta}-0 \int_{1}^{\infty} x^{-\alpha / \beta} s^{-\alpha} d s=x^{-\alpha / \beta}
\end{aligned}
$$

since $T / \zeta^{\leftarrow}(b(T) x) \rightarrow \infty$ as $T \rightarrow \infty$, see (3.11). Hence, for every fixed $t$,

$$
T P\left(\zeta\left(J_{k}^{(T t)}\right)>b(T) x\right) \rightarrow x^{-\alpha / \beta}
$$

as $T \rightarrow \infty$.

\section{Weak Convergence in $D[0, \infty)$}

In the previous section, we showed that the finite dimensional distributions of the scaled process $\left(A(T t)-\lambda \mu_{\zeta} T t\right) / b(T)$ converge as $T \rightarrow \infty$, to those of a skewed $\alpha / \beta$ stable Lévy motion $X_{\alpha / \beta}(t)$. For telecommunications applications such as the heavy traffic theorem discussed in the next section, we need convergence in the stronger functional sense in a suitable topology. When the limit process has almost surely continuous sample paths, one typically uses the Skorohod $J_{1}$-topology, or if possible the uniform topology. As remarked in [17, 30,31, 29], it is not possible for a family of continuous processes $X_{T} \in C(0, \infty]$ to converge in the $J_{1}$ sense to a limit $X$ that is discontinuous with positive probability.

Hence, we investigate convergence in a weaker topology than $J_{1}$ and show in this section that $\left.\left.\left(A(T t)-\lambda \mu_{\zeta} T t\right)\right)\right) / b(T)$ converges to $X_{\alpha / \beta}(t)$ in $D[0, \infty)$ endowed with the $M_{1}$-topology.

The investigation of functional limit theorems for processes with paths in $D[0,1]$ was started by Skorohod [27], who introduced four topologies on $D[0,1]$, which he called $J_{1}, J_{2}, M_{1}$ and $M_{2}$. For defining the $M_{1}$ and $J_{1}$ topologies, we need the functions

$$
J\left(x_{1}, x_{2}, x_{3}\right)=\min \left\{\left|x_{1}-x_{2}\right|,\left|x_{3}-x_{2}\right|\right\}
$$


and

$$
\begin{aligned}
M\left(x_{1}, x_{2}, x_{3}\right) & =\text { the distance from } x_{2} \text { to the line segment joining } x_{1} \text { and } x_{3} \\
& = \begin{cases}0, & \text { if } x_{2} \in\left[x_{1} \wedge x_{3}, x_{1} \vee x_{3}\right], \\
J\left(x_{1}, x_{2}, x_{3}\right), & \text { otherwise. }\end{cases}
\end{aligned}
$$

The function $M\left(x_{1}, x_{2}, x_{3}\right)$ is 0 if $x_{2}$ is in the line segment with endpoints $x_{1}$ and $x_{3}$ and is the minimum distance of $x_{2}$ to the endpoints of the segment otherwise.

Letting $H$ stand for either $J$ or $M$, we define the $H$-oscillation (see $[27,2,3]$ ) of a function $z(t)$ on $[0,1]$ as

$$
\omega_{\delta}^{H}(Z):=\sup _{\substack{0 \leq t_{1} \leq t \leq t_{2} \leq 1 \\ 0 \leq t_{2}-t_{1} \leq \delta}} H\left(z\left(t_{1}\right), z\left(t_{2}\right), z\left(t_{3}\right)\right) .
$$

We need the following criterion from [27], Theorems 3.2.1 and 3.2.2, which can also be found as Proposition 2 in [3]:

Proposition 4.1. Let $Z_{n}(t)$ be processes in $D[0,1]$, whose finite dimensional distributions converge to those of a process $Z(t)$, which is almost surely continuous at $t=0$ and $t=1$. Let $H$ stand for either $J$ or $M$. Then weak $H_{1}$-convergence holds if and only if for every $\epsilon>0$

$$
\lim _{\delta \rightarrow 0} \limsup _{n \rightarrow \infty} P\left(\omega_{\delta}^{H}\left(Z_{n}\right)>\epsilon\right)=0
$$

Remark 4.1. An analogous result holds for processes in $D[0, b], b$ arbitrary, provided the limit process $Z(t)$ is a.s. continuous at $t=0$ and at $t=b$.

As mentioned in [31], Section 8, p.20, convergence in $D[0, \infty)$ is equivalent to convergence in $D[0, b]$ for all $b>0$. Let

$$
Z_{T}(t)=\frac{\left.A(T t)-\lambda \mu_{\zeta} T t\right)}{b(T)}
$$

By Proposition 4.1, we need to show

$$
\lim _{\delta \rightarrow 0} \limsup _{T \rightarrow \infty} P\left(\omega_{\delta}^{M}\left(Z_{T}\right)>\epsilon\right)=0 .
$$

We will do this in the following Theorem.

Theorem 4.1. For $0 \leq t_{1} \leq t \leq t_{2} \leq b$, there exists a $T_{1}$ depending on $\left(t_{2}-t_{1}\right)$ such that for $T>T_{1}$, we have

$$
P\left(M\left(Z_{T}\left(t_{1}\right), Z_{T}(t), Z_{T}\left(t_{2}\right)\right)>\epsilon\right) \leq \lambda^{2} C^{2} \epsilon^{2(\alpha / \beta+\eta)}\left(t_{2}-t_{1}\right)^{2} .
$$

Therefore

$$
\limsup _{T \rightarrow \infty} P\left(\omega_{\delta}^{M}\left(Z_{T}\right)>\epsilon\right) \leq K \lambda^{2} C^{2} \epsilon^{2(\alpha / \beta+\eta)} \delta
$$

and $Z_{T}$ converges in $\left(D[0, \infty), M_{1}\right)$.

Proof. If $t_{1}=t=t_{2}$, there is nothing to prove. By (4.3):

$$
\begin{gathered}
P\left(M\left(Z_{T}\left(t_{1}\right), Z_{T}(t), Z_{T}\left(t_{2}\right)\right)>\epsilon\right) \\
=P\left[Z_{T}(t)>Z_{T}\left(t_{2}\right) \vee Z_{T}\left(t_{1}\right)+\epsilon\right]+P\left[Z_{T}(t)<Z_{T}\left(t_{2}\right) \wedge Z_{T}\left(t_{1}\right)-\epsilon\right] \\
=P\left(Z_{T}(t)-Z_{T}\left(t_{1}\right)>\epsilon, Z_{T}\left(t_{2}\right)-Z_{T}(t) \leq-\epsilon\right) \\
\quad+P\left(Z_{T}(t)-Z_{T}\left(t_{1}\right) \leq-\epsilon, Z_{T}\left(t_{2}\right)-Z_{T}(t)>\epsilon\right) .
\end{gathered}
$$


Consider the first term on the right hand side of (4.7):

$$
\begin{aligned}
P\left(Z_{T}(t)-Z_{T}\left(t_{1}\right)>\epsilon, Z_{T}\left(t_{2}\right)-Z_{T}(t) \leq-\epsilon\right) & \\
= & P\left(\frac{A(T t)-A\left(T t_{1}\right)-\lambda \mu_{\zeta} T\left(t-t_{1}\right)}{b(T)}>\epsilon, \frac{A\left(T t_{2}\right)-A(T t)-\lambda \mu_{\zeta} T\left(t_{2}-t\right)}{b(T)} \leq-\epsilon\right) \\
= & P\left(\frac{A_{1}(T t)-A_{1}\left(T t_{1}\right)+A_{2}(T t)-A_{2}\left(T t_{1}\right)-\lambda \mu_{\zeta} T\left(t-t_{1}\right)}{b(T)}>\epsilon,\right. \\
& \left.\frac{A_{1}\left(T t_{2}\right)-A_{1}(T t)+A_{2}\left(T t_{2}\right)-A_{2}(T t)-\lambda \mu_{\zeta} T\left(t_{2}-t\right)}{b(T)} \leq-\epsilon\right) .
\end{aligned}
$$

Write

$$
\begin{aligned}
A_{1}(T t)-A_{1}\left(T t_{1}\right) & =\iint_{\substack{0 \leq u \leq T t_{1} \\
T t_{1} \leq u+y \leq T t}} \zeta(u) M(d u d y)+\iint_{\substack{T t_{1} \leq u \leq T t \\
0 \leq u+y \leq T t}} \zeta(u) M(d u d y) \\
& =: A_{2}^{\prime}+A_{1}^{\prime}\left(T\left(t-t_{1}\right),\right. \\
A_{1}\left(T t_{2}\right)-A_{1}(T t) & =\iint_{\substack{0 \leq u \leq T t \\
T t \leq u+y \leq T t_{2}}} \zeta(u) M(d u d y)+\iint_{\substack{T t \leq u \leq T t_{2} \\
0 \leq u+y \leq T t_{2}}} \zeta(u) M(d u d y) \\
& =: A_{2}^{\prime \prime}+A_{1}^{\prime \prime}\left(T\left(t-t_{1}\right) .\right.
\end{aligned}
$$

The previous probability is

$$
\begin{aligned}
P[ & \frac{A_{1}^{\prime}\left(T\left(t-t_{1}\right)-\lambda \mu_{\zeta}\left(T\left(t-t_{1}\right)\right.\right.}{b(T)}+\frac{A_{2}^{\prime}+A_{2}(T t)-A_{2}\left(T t_{1}\right)}{b(T)}>\epsilon, \\
& \left.\frac{A_{1}^{\prime \prime}\left(T\left(t_{2}-t\right)-\lambda \mu_{\zeta}\left(T\left(t_{2}-t\right)\right.\right.}{b(T)}+\frac{A_{2}^{\prime \prime}+A_{2}\left(T t_{2}\right)-A_{2}(T t)}{b(T)}<-\epsilon\right]
\end{aligned}
$$

and using $(A \cup B) \cap(C \cup D)=A C \cup A D \cup B C \cup B D$ and subadditivity we get the bound

$$
\begin{aligned}
P & \left(\frac{A_{1}^{\prime}\left(T\left(t-t_{1}\right)\right)-\lambda \mu_{\zeta} T\left(t-t_{1}\right)}{b(T)}>\epsilon / 2, \frac{A_{1}^{\prime \prime}\left(T\left(t_{2}-t\right)\right)-\lambda \mu_{\zeta} T\left(t_{2}-t\right)}{b(T)} \leq-\epsilon / 2\right) \\
& +P\left(\frac{A_{1}^{\prime}\left(T\left(t-t_{1}\right)\right)-\lambda \mu_{\zeta} T\left(t-t_{1}\right)}{b(T)}>\epsilon / 2, \frac{A_{2}^{\prime \prime}+A_{2}\left(T t_{2}\right)-A_{2}(T t)}{b(T)} \leq-\epsilon / 2\right) \\
& +P\left(\frac{A_{2}^{\prime}+A_{2}(T t)-A_{2}\left(T t_{1}\right)}{b(T)}>\epsilon / 2, \frac{A_{1}^{\prime \prime}\left(T\left(t_{2}-t\right)\right)-\lambda \mu_{\zeta} T\left(t_{2}-t\right)}{b(T)} \leq-\epsilon / 2\right) \\
& +P\left(\frac{A_{2}^{\prime}+A_{2}(T t)-A_{2}\left(T t_{1}\right)}{b(T)}>\epsilon / 2, \frac{A_{2}^{\prime \prime}+A_{2}\left(T t_{2}\right)-A_{2}(T t)}{b(T)} \leq-\epsilon / 2\right) .
\end{aligned}
$$

Here $A_{1}^{\prime}$ and $A_{1}^{\prime \prime}$ are independent and identically distributed, as are $A_{2}^{\prime}$ and $A_{2}^{\prime \prime}$. Recalling we work on $[0, b]$, we may use Markov's inequality on the last three terms to get the bound

$$
\begin{aligned}
3\left(\frac{E\left(A_{2}^{\prime}\right)+E\left(A_{2}^{\prime \prime}\right)+2 E\left(A_{2}(T b)\right)}{b(T) \epsilon / 2}\right) & =\frac{6}{\epsilon}\left(\frac{E\left(A_{2}^{\prime}\right)+E\left(A_{2}^{\prime \prime}\right)+2 E\left(A_{2}(T b)\right)}{b(T)}\right) \\
& \sim(\text { const }) \frac{T \zeta(T) \bar{F}(T)}{b(T)} \rightarrow 0
\end{aligned}
$$

as $T \rightarrow \infty$, by (3.24). The constant is of the form (cf. (3.33), (3.23))

$$
\frac{6}{\epsilon}\left(c\left(t_{1}, t\right)+c\left(t, t_{2}\right)+k(b)\right)
$$


where $k(b)$ depends only on $b$.

For the first probability in (4.8), we can factor to get

$$
\begin{aligned}
& P\left(\frac{A_{1}^{\prime}\left(T\left(t-t_{1}\right)\right)-\lambda \mu_{\zeta} T\left(t-t_{1}\right)}{b(T)}>\epsilon / 2, \frac{A_{1}^{\prime \prime}\left(T\left(t_{2}-t\right)\right)-\lambda \mu_{\zeta} T\left(t_{2}-t\right)}{b(T)} \leq-\epsilon / 2\right) \\
& \quad=P\left(\frac{A_{1}^{\prime}\left(T\left(t-t_{1}\right)\right)-\lambda \mu_{\zeta} T\left(t-t_{1}\right)}{b(T)}>\epsilon / 2\right) P\left(\frac{A_{1}^{\prime \prime}\left(T\left(t_{2}-t\right)\right)-\lambda \mu_{\zeta} T\left(t_{2}-t\right)}{b(T)} \leq-\epsilon / 2\right) .
\end{aligned}
$$

Consider the first factor

$(4.10)=A+B$.

$$
\begin{aligned}
& P\left(\frac{A_{1}^{\prime}\left(T\left(t-t_{1}\right)\right)-\lambda \mu_{\zeta} T\left(t-t_{1}\right)}{b(T)}>\epsilon\right)=P\left(\frac{\sum_{k=1}^{P_{1}\left(T\left(t-t_{1}\right)\right)} \zeta\left(J_{k}^{(T)}\right)-\lambda \mu_{\zeta} T\left(t-t_{1}\right)}{b(T)}>\epsilon\right) \\
& \quad=P\left(\frac{\sum_{k=1}^{P_{1}\left(T\left(t-t_{1}\right)\right)}\left(\zeta\left(J_{k}^{(T)}\right)-\mu_{\zeta}^{(T)}\right)}{b(T)}+\frac{P_{1}\left(T\left(t-t_{1}\right)\right) \mu_{\zeta}^{(T)}-\lambda \mu_{\zeta} T\left(t-t_{1}\right)}{b(T)}>\epsilon\right) \\
& \quad \leq P\left(\frac{\sum_{k=1}^{P_{1}\left(T\left(t-t_{1}\right)\right)}\left(\zeta\left(J_{k}^{(T)}\right)-\mu_{\zeta}^{(T)}\right)}{b(T)}>\epsilon / 2\right)+P\left(\frac{P_{1}\left(T\left(t-t_{1}\right)\right) \mu_{\zeta}^{(T)}-\lambda \mu_{\zeta} T\left(t-t_{1}\right)}{b(T)}>\epsilon / 2\right)
\end{aligned}
$$

Now

$$
\begin{aligned}
B & \leq P\left(\left|\left(\frac{P_{1}\left(T\left(t-t_{1}\right)\right)-\lambda T\left(t-t_{1}\right)}{b(T)}\right) \mu_{\zeta}\right|>\epsilon / 4\right)+P\left(\frac{\left(\mu_{\zeta}-\mu_{\zeta}^{(T)}\right) P_{1}\left(T\left(t-t_{1}\right)\right)}{b(T)}>\epsilon / 4\right) \\
& =B I+B I I .
\end{aligned}
$$

Consider $B I$ : From the central limit theorem for Poisson variables, we know that

$$
B_{T\left(t-t_{1}\right)}:=\frac{P_{1}\left(T\left(t-t_{1}\right)\right)-\lambda \hat{F}\left(T\left(t-t_{1}\right)\right)}{\sqrt{\lambda \hat{F}\left(T\left(t-t_{1}\right)\right)}} \Rightarrow B(1) \sim \mathcal{N}(0,1)
$$

and

$$
E\left|B_{T\left(t-t_{1}\right)}\right| \leq E\left(B_{T\left(t-t_{1}\right)}^{2}\right)^{1 / 2}=1
$$

so

$$
\begin{aligned}
B I & \leq \frac{4}{\epsilon} E\left|\frac{P_{1}\left(T\left(t-t_{1}\right)\right)-\lambda T\left(t-t_{1}\right)}{b(T)} \mu_{\zeta}\right| \\
& \leq \frac{4}{\epsilon}\left(\mu_{\zeta} \frac{E\left|P_{1}\left(T\left(t-t_{1}\right)\right)-\lambda \hat{F}\left(T\left(t-t_{1}\right)\right)\right|}{b(T)}+\mu_{\zeta}\left|\frac{\lambda\left(\hat{F}\left(T\left(t-t_{1}\right)\right)-T\left(t-t_{1}\right)\right)}{b(T)}\right|\right) \\
& \leq \frac{4}{\epsilon}\left(\frac{\sqrt{\lambda \hat{F}\left(T\left(t-t_{1}\right)\right)}}{b(T)} \mu_{\zeta}+\left|\frac{\lambda\left(\hat{F}\left(T\left(t-t_{1}\right)\right)-T\left(t-t_{1}\right)\right)}{b(T)} \mu_{\zeta}\right|\right) \\
& \leq \frac{4}{\epsilon}\left(\frac{\sqrt{\lambda \hat{F}\left(T\left(t-t_{1}\right)\right.}}{b(T)} \mu_{\zeta}+\frac{\lambda \mu_{\zeta} \mu}{b(T)}\right) \rightarrow 0,
\end{aligned}
$$

(see the argument between (3.28) and (3.29)) as $T \rightarrow \infty$, since $b(T)$ is regularly varying with index $\beta / \alpha>1 / 2$. 
To handle $B I I$, we argue similarly:

$$
\begin{aligned}
\frac{\left(\mu_{\zeta}-\mu_{\zeta}^{(T)}\right) P_{1}\left(T\left(t-t_{1}\right)\right)}{b(T)} & =\frac{\left(\mu_{\zeta}-\mu_{\zeta}^{(T)}\right) \lambda \hat{F}\left(T\left(t-t_{1}\right)\right)}{b(T)}+\frac{\left(\mu_{\zeta}-\mu_{\zeta}^{(T)}\right)\left(P_{1}\left(T\left(t-t_{1}\right)\right)-\lambda \hat{F}\left(T\left(t-t_{1}\right)\right)\right)}{b(T)} \\
& =T I I_{1}+T I I_{2} .
\end{aligned}
$$

From (3.26),

$$
\begin{aligned}
T I I_{1} & =(\text { const })\left(t-t_{1}\right) \frac{\zeta(T) \bar{F}(T) T}{b(T)}+\lambda \frac{\mu_{\zeta} \mu}{b(T)} \\
& \leq\left(\text { const }_{1}\right)\left(t_{2}-t_{1}\right) \frac{\zeta(T) \bar{F}(T) T}{b(T)} \rightarrow 0
\end{aligned}
$$

as $T \rightarrow \infty$ from (3.24). For $T I I_{2}$ we have from (4.11)

$$
\begin{aligned}
P\left[T I I_{2}>\epsilon\right] & \leq \frac{1}{\epsilon} E\left|B_{T\left(t-t_{1}\right)}\right| \mu_{\zeta} \cdot \frac{\sqrt{\lambda \hat{F}\left(T\left(t-t_{1}\right)\right.}}{b(T)} \\
& \leq \frac{1}{\epsilon} \mu_{\zeta} \frac{\sqrt{\lambda \hat{F}\left(T\left(t_{2}-t_{1}\right)\right.}}{b(T)} \rightarrow 0,
\end{aligned}
$$

as $T \rightarrow \infty$. This concludes the discussion of $B$.

Next, we consider $A$. We have

$$
\sum_{k=1}^{P_{1}\left(T\left(t-t_{1}\right)\right.}\left|\frac{\zeta\left(J_{k}^{(T)}\right)-\mu_{\zeta}^{(T)}}{b(T)}\right| \leq \sum_{k=1}^{\left[\lambda \hat{F}\left(T\left(t-t_{1}\right)\right]\right.}\left|\frac{\zeta\left(J_{k}^{(T)}\right)-\mu_{\zeta}^{(T)}}{b(T)}\right|+\sum_{k=\left[\lambda \hat{F}\left(T\left(t-t_{1}\right)\right]+1\right.}^{P_{1}\left(T\left(t-t_{1}\right)\right.}\left|\frac{\zeta\left(J_{k}^{(T)}\right)-\mu_{\zeta}^{(T)}}{b(T)}\right|
$$

and therefore

$$
\begin{aligned}
A & \leq P\left(\sum_{k=1}^{\left[\lambda \hat{F}\left(T\left(t-t_{1}\right)\right)\right]}\left|\frac{\left(\zeta\left(J_{k}^{(T)}\right)-\mu_{\zeta}^{(T)}\right)}{b(T)}\right|>\epsilon / 2\right)+P\left(\sum_{k=1}^{\left|\left[\lambda \hat{F}\left(T\left(t-t_{1}\right)\right)\right]-P_{1}\left(T\left(t-t_{1}\right)\right)\right|}\left|\frac{\zeta\left(J_{k}^{(T)}\right)-\mu_{\zeta}^{(T)}}{b(T)}\right|>\epsilon / 2\right) \\
& =: A I+A I I .
\end{aligned}
$$

Now $A I I$ can be bounded using Markov's inequality, and the fact that $P_{1}\left(T\left(t-t_{1}\right)\right)$ is a Poisson random variable:

$$
\begin{aligned}
A I I & \leq P\left(\left|\sum_{k=1}^{\left|\left[\lambda \hat{F}\left(T\left(t-t_{1}\right)\right)\right]-P_{1}\left(T\left(t-t_{1}\right)\right)\right|}\right| \frac{\zeta\left(J_{k}^{(T)}\right)-\mu_{\zeta}^{(T)}}{b(T)} \mid>\epsilon / 2\right) \\
& \leq(2 / \epsilon) E\left(\sum_{k=1}^{\left|\left[\lambda \hat{F}\left(T\left(t-t_{1}\right)\right)\right]-P_{1}\left(T\left(t-t_{1}\right)\right)\right|}\left|\frac{\zeta\left(J_{k}^{(T)}\right)-\mu_{\zeta}^{(T)}}{b(T)}\right|\right) \\
& \leq(2 / \epsilon) \mu_{\zeta} \frac{E\left|\left[\lambda \hat{F}\left(T\left(t-t_{1}\right)\right)\right]-P_{1}\left(T\left(t-t_{1}\right)\right)\right|}{b(T)} \\
& \leq(2 / \epsilon) \mu_{\zeta} \frac{\sqrt{\lambda \hat{F}\left(T\left(t_{2}-t_{1}\right)\right)}}{b(T)} \rightarrow 0
\end{aligned}
$$

as $T \rightarrow \infty$, as seen before. 
Now we deal with $A I$. To bound this probability, we argue as in the proof of Lemma 2 of [2]: Pick $\eta>0$ such that $\alpha / \beta-\eta>1$. Let

$$
Y_{k, T}=\frac{\left(\zeta\left(J_{k}^{(T)}\right)-\mu_{\zeta}^{(T)}\right)}{b(T)}
$$

and define

$$
\begin{array}{cl}
Y_{k, T}^{\leq}=Y_{k, T} 1_{\left(\left|Y_{k, T}\right| \leq 1\right)}, & Y_{k, T}^{>}=Y_{k, T} 1_{\left(\left|Y_{k, T}\right|>1\right)} \\
\bar{Y}_{k, T}^{\leq}=Y_{k, T}^{\leq}-E\left(Y_{k, T}^{\leq}\right), & \bar{Y}_{k, T}^{>}=Y_{k, T}^{>}-E\left(Y_{k, T}^{>}\right)
\end{array}
$$

Now

$$
P\left(\sum_{k=1}^{\left[\lambda \hat{F}\left(T\left(t-t_{1}\right)\right)\right]}\left|Y_{k, T}\right|>\epsilon\right) \leq P\left(\sum_{k=1}^{\left[\lambda \hat{F}\left(T\left(t-t_{1}\right)\right)\right]}\left|\bar{Y}_{k, T}^{\leq}\right|>\epsilon / 2\right)+P\left(\sum_{k=1}^{\left[\lambda \hat{F}\left(T\left(t-t_{1}\right)\right)\right]}\left|\bar{Y}_{k, T}^{>}\right|>\epsilon / 2\right) .
$$

Using Chebychev's inequality with $p=\alpha / \beta-\eta$, followed by the von Bahr-Esseen inequality, which says that $E\left|\sum_{j=1}^{m} X_{j}\right|^{p} \leq 2 \sum_{j=1}^{m} E\left|X_{j}\right|^{p}$ if $1 \leq p \leq 2$, and $X_{j}$ a martingale difference sequence, we get

$$
\begin{aligned}
P\left(\sum_{k=1}^{\left[\lambda \hat{F}\left(T\left(t-t_{1}\right)\right)\right]}\left|\bar{Y}_{k, T}^{>}\right|>\epsilon / 2\right) & \leq(\epsilon / 2)^{-(\alpha / \beta-\eta)} E\left(\sum_{k=1}^{\left[\lambda \hat{F}\left(T\left(t-t_{1}\right)\right)\right]}\left|\bar{Y}_{k, T}^{>}\right|\right)^{[\alpha / \beta-\eta)} \\
& \leq 2(\epsilon / 2)^{-(\alpha / \beta-\eta)}\left[\lambda \hat{F}\left(T\left(t-t_{1}\right)\right)\right] E\left|\bar{Y}_{k, T}^{>}\right|^{\alpha / \beta-\eta} .
\end{aligned}
$$

Similarly, but using Chebychev's inequality with $p=\alpha / \beta+\eta$,

$$
P\left(\sum_{k=1}^{\left[\lambda \hat{F}\left(T\left(t-t_{1}\right)\right)\right]}\left|\bar{Y}_{k, T}^{\leq}\right|>\epsilon / 2\right) \leq 2(\epsilon / 2)^{-(\alpha / \beta+\eta)}\left[\lambda \hat{F}\left(T\left(t-t_{1}\right)\right)\right] E\left|\bar{Y}_{k, T}^{\leq}\right|^{\alpha / \beta-\eta} .
$$

By Jensen's inequality,

$$
E\left|Y_{k, T}^{\leq}-E\left(Y_{k, T}^{\leq}\right)\right|^{\alpha / \beta+\eta} \leq 2^{\alpha / \beta+\eta-1}\left\{E\left|Y_{k, T}^{\leq}\right|^{\alpha / \beta+\eta}+\left|E Y_{k, T}^{\leq}\right|^{\alpha / \beta+\eta}\right\} \leq 2^{\alpha / \beta+\eta} E\left|Y_{k, T}^{\leq}\right|^{\alpha / \beta+\eta} .
$$

Similarly,

$$
E\left|Y_{k, T}^{>}-E Y_{k, T}^{>}\right|^{\alpha / \beta-\eta} \leq 2^{\alpha / \beta-\eta} E\left|Y_{k, T}^{>}\right|^{\alpha / \beta-\eta} .
$$

Using Lemma 1 of [1], it follows that

$$
\left\{\sup _{T}\left(T E\left|\bar{Y}_{k, T}^{\leq}\right|^{\alpha / \beta+\eta}\right)\right\} \vee \underset{T}{\sup }\left(T E\left|\bar{Y}_{k, T}^{>}\right|^{\alpha / \beta-\eta}\right\} \leq C<\infty
$$

and thus

$$
P\left(\sum_{k=1}^{\left[\lambda \hat{F}\left(T\left(t-t_{1}\right)\right)\right]}\left|Y_{k, T}\right|>\epsilon\right) \leq C \epsilon^{-(\alpha / \beta+\eta)} \frac{\left[\lambda \hat{F}\left(T\left(t-t_{1}\right)\right)\right]}{T} .
$$

Now $\hat{F}\left(T\left(t-t_{1}\right)\right) \leq \hat{F}\left(T\left(t_{2}-t_{1}\right)\right) \sim T\left(t_{2}-t_{1}\right)$, so for $T$ large enough (depending on $\left.t_{2}-t_{1}\right)$,

$$
A I \leq P\left(\sum_{k=1}^{\left[\lambda \hat{F}\left(T\left(t-t_{1}\right)\right)\right]}\left|Y_{k, T}\right|>\epsilon\right) \leq 2 \lambda C \epsilon^{-(\alpha / \beta+\eta)}\left(t_{2}-t_{1}\right) .
$$


Summarizing (4.10), (4.12), (4.13), (4.14), (4.18), we have for $t_{2}-t_{1}<\delta$ and $T$ large enough, depending on $t_{2}-t_{1}$ that

$$
\begin{aligned}
& P\left(\left|\frac{A^{\prime}\left(T\left(t-t_{1}\right)\right)-\lambda \mu_{\zeta} T\left(t-t_{1}\right)}{b(T)}\right|>\epsilon\right) \\
& \quad \leq P\left(\sum_{k=1}^{\left[\lambda \hat{F}\left(T\left(t-t_{1}\right)\right)\right]}\left|\frac{\zeta\left(J_{k}^{(T)}\right)-\mu_{\zeta}^{(T)}}{b(T)}\right|>\epsilon / 2\right)+\left(\frac{16}{\epsilon}\right)\left(\frac{\lambda \mu_{\zeta} \mu}{b(T)}+\mu_{\zeta} \frac{\sqrt{\lambda \hat{F}(T \delta)}}{b(T)}\right) \\
& \quad \leq 2 \lambda M \epsilon^{-(\alpha / \beta+\eta)}\left(t_{2}-t_{1}\right)+\left(\frac{16}{\epsilon}\right)\left(\frac{\lambda \mu_{\zeta} \mu}{b(T)}+\mu_{\zeta} \frac{\sqrt{\lambda \hat{F}(T \delta)}}{b(T)}\right)
\end{aligned}
$$

Recall (4.7)

$$
\begin{aligned}
P\left(M\left(Z_{T}\left(t_{1}\right), Z_{T}(t), Z_{T}\left(t_{2}\right)\right)>\epsilon\right) & =P\left(Z_{T}(t)-Z_{T}\left(t_{1}\right)>\epsilon, Z_{T}\left(t_{2}\right)-Z_{T}(t) \leq-\epsilon\right) \\
& +P\left(Z_{T}(t)-Z_{T}\left(t_{1}\right) \leq-\epsilon, Z_{T}\left(t_{2}\right)-Z_{T}(t)>\epsilon\right)
\end{aligned}
$$

and from (4.8) and (4.9)

$$
\begin{aligned}
& P\left(Z_{T}(t)-Z_{T}\left(t_{1}\right)>\epsilon, Z_{T}\left(t_{2}\right)-Z_{T}(t) \leq-\epsilon\right) \\
& \leq P\left(\frac{A_{1}^{\prime}\left(T\left(t-t_{1}\right)\right)-\lambda \mu_{\zeta} T\left(t-t_{1}\right)}{b(T)}>\epsilon, \frac{A_{1}^{\prime \prime}\left(T\left(t_{2}-t\right)\right)-\lambda \mu_{\zeta} T\left(t_{2}-t\right)}{b(T)} \leq-\epsilon\right)+C_{1} \frac{T \zeta(T) \bar{F}(T)}{b(T)} \\
& =P\left(\frac{A_{1}^{\prime}\left(T\left(t-t_{1}\right)\right)-\lambda \mu_{\zeta} T\left(t-t_{1}\right)}{b(T)}>\epsilon\right) P\left(\frac{A_{1}^{\prime \prime}\left(T\left(t_{2}-t\right)\right)-\lambda \mu_{\zeta} T\left(t_{2}-t\right)}{b(T)} \leq-\epsilon\right)+C_{1} \frac{T \zeta(T) \bar{F}(T)}{b(T)} \\
& \leq P\left(\left|\frac{A_{1}^{\prime}\left(T\left(t-t_{1}\right)\right)-\lambda \mu_{\zeta} T\left(t-t_{1}\right)}{b(T)}\right|>\epsilon\right) P\left(\left|\frac{A_{1}^{\prime \prime}\left(T\left(t_{2}-t\right)\right)-\lambda \mu_{\zeta} T\left(t_{2}-t\right)}{b(T)}\right|>\epsilon\right)+C_{1} \frac{T \zeta(T) \bar{F}(T)}{b(T)} .
\end{aligned}
$$

Similarly,

$$
\begin{aligned}
& P\left(Z_{T}(t)-Z_{T}\left(t_{1}\right) \leq-\epsilon, Z_{T}\left(t_{2}\right)-Z_{T}(t)>\epsilon\right) \\
& \leq P\left(\left|\frac{A_{1}^{\prime}\left(T\left(t-t_{1}\right)\right)-\lambda \mu_{\zeta} T\left(t-t_{1}\right)}{b(T)}\right|>\epsilon\right) P\left(\left|\frac{A_{1}^{\prime \prime}\left(T\left(t_{2}-t\right)\right)-\lambda \mu_{\zeta} T\left(t_{2}-t\right)}{b(T)}\right|>\epsilon\right)+C_{1} \frac{T \zeta(T) \bar{F}(T)}{b(T)} .
\end{aligned}
$$

So since $A_{1}^{\prime} \stackrel{d}{=} A_{1}^{\prime \prime}$,

$$
\begin{gathered}
P\left(M\left(Z_{t}\left(t_{1}\right), Z_{T}(t), Z_{T}\left(t_{2}\right)\right)>\epsilon\right) \\
\leq 2\left(2 \lambda C \epsilon^{-(\alpha / \beta+\eta)}\left(t_{2}-t_{1}\right)+\left(\frac{16}{\epsilon}\right)\left(\frac{\lambda \mu_{\zeta} \mu}{b(T)}+\mu_{\zeta} \frac{\sqrt{\lambda \hat{F}(T \delta)}}{b(T)}\right)^{2}+2 C_{1} \frac{T \zeta(T) \bar{F}(T)}{b(T)}\right. \\
=8 \lambda^{2} C^{2} \epsilon^{-2(\alpha / \beta+\eta)}\left(t_{2}-t_{1}\right)^{2}+4\left(\frac{16}{\epsilon}\right)\left(\frac{\lambda \mu_{\zeta} \mu}{b(T)}+\mu_{\zeta} \frac{\sqrt{\lambda \hat{F}(T \delta)}}{b(T)}\right) 2 \lambda C \epsilon^{-(\alpha / \beta+\eta)}\left(t_{2}-t_{1}\right) \\
\quad+2\left(\left(\frac{16}{\epsilon}\right)^{2}\left(\frac{\lambda \mu_{\zeta} \mu}{b(T)}+\mu_{\zeta} \frac{\sqrt{\lambda \hat{F}(T \delta)}}{b(T)}\right)^{2}+2 C_{1} \frac{T \zeta(T) \bar{F}(T)}{b(T)} .\right.
\end{gathered}
$$

For $T>T_{1}$, with $T_{1}$ depending on $t_{2}-t_{1}, \delta, \alpha / \beta+\eta$, we have

$$
P\left(M\left(Z_{T}\left(t_{1}\right), Z_{T}(t), Z_{T}\left(t_{2}\right)\right)>\epsilon\right) \leq \lambda^{2} C^{2} \epsilon^{-2(\alpha / \beta+\eta)}\left(t_{2}-t_{1}\right)^{2}
$$


Let $Z_{T, n}(t)$ be the step function built from the values of $Z_{T}(t)$ at $t=i / 2^{n}, i=1, \ldots, 2^{n}$. For $T>T_{1}$, also

$$
P\left(M\left(Z_{T, n}\left(t_{1}\right), Z_{T, n}(t), Z_{T, n}\left(t_{2}\right)\right)>\epsilon\right) \leq \lambda^{2} C^{2} \epsilon^{-2(\alpha / \beta+\eta)}\left(t_{2}-t_{1}\right)^{2},
$$

and thus, by Theorem 2 of [2], we have

$$
P\left(\sup _{t_{1} \leq c \leq d \leq e \leq t_{2}} M\left(Z_{T, n}(c), Z_{T, n}(d), Z_{T, n}(e)\right)>\epsilon\right) \leq K \lambda^{2} C^{2} \epsilon^{-2(\alpha / \beta+n)}\left(t_{2}-t_{1}\right)^{2} .
$$

Now, as in the proof of Lemma 1 of [2]: let $m=\left[\delta^{-1}\right]$ and partition $[0, b]$ with $t_{i}, i=0,1, \ldots, m+1$, where $t_{0}=0, t_{m+1}=b$, and $t_{i+1}-t_{i}=\delta, i=0,1, \ldots, m-1$. The event $\left\{\omega_{\delta}^{M}\left(Z_{T, n}\right) \geq \epsilon\right\}$ implies $\left\{\max _{i=0, \ldots, m-1} \sup _{t_{1} \leq c \leq d \leq e \leq t_{2}} M\left(Z_{T, n}(c), Z_{T, n}(d), Z_{T, n}(e)\right) \geq \epsilon\right\}$.

Indeed, if there exist points $c \leq d \leq e$ such that $e-c \leq \delta$, then these points belong to some interval $\left[t_{i}, t_{i+2}\right]$, so that $M\left(Z_{T, n}(c), Z_{T, n}(d), Z_{T, n}(e)\right) \geq \epsilon$ implies

$$
\sup _{t_{i} \leq c \leq d \leq e \leq t_{i+2}} M\left(Z_{T, n}(c), Z_{T, n}(d), Z_{T, n}(e)\right) \geq \epsilon .
$$

Therefore, for $T>T_{1}$, we have

$$
\begin{aligned}
P\left(\omega_{\delta}^{M}\left(Z_{T, n}\right) \geq \epsilon\right) & \leq \sum_{i=0}^{m-1} P\left(\sup _{t_{i} \leq c \leq d \leq e \leq t_{i+2}} M\left(Z_{T, n}(c), Z_{T, n}(d), Z_{T, n}(e)\right)>\epsilon\right) \\
& \leq K \lambda^{2} C^{2} \epsilon^{2(\alpha / \beta+\eta)}\left(t_{2}-t_{1}\right)^{2} \\
& \leq\left[\delta^{-1}\right] K \lambda^{2} C^{2} \epsilon^{2(\alpha / \beta+\eta)}(2 \delta)^{2} \\
& \leq K^{\prime} \lambda^{2} C^{2} \epsilon^{2(\alpha / \beta+\eta)} \delta .
\end{aligned}
$$

Since almost all paths of $Z_{T}$ are right continuous and $\omega_{\delta}^{M}\left(Z_{T, n}\right) \rightarrow \omega_{\delta}^{M}\left(Z_{T}\right)$ a.s., we have

$$
P\left(\omega_{\delta}^{M}\left(Z_{T}\right) \geq \epsilon\right)=\lim _{n \rightarrow \infty} P\left(\omega_{\delta}^{M}\left(Z_{T, n}\right) \geq \epsilon\right),
$$

and hence, for $T>T_{1}$

$$
P\left(\omega_{\delta}^{M}\left(Z_{T}\right) \geq \epsilon\right) \leq K^{\prime} \lambda^{2} C^{2} \epsilon^{2(\alpha / \beta+\eta)} \delta
$$

This implies

$$
\limsup _{T \rightarrow \infty} P\left(\omega_{\delta}^{M}\left(Z_{T}\right)>\epsilon\right) \leq K^{\prime} \lambda^{2} C^{2} \epsilon^{2(\alpha / \beta+\eta)} \delta
$$

and thus

$$
\lim _{\delta \rightarrow 0} \limsup _{T \rightarrow \infty} P\left(\omega_{\delta}^{M}\left(Z_{T}\right) \geq \epsilon\right) \leq \lim _{\delta \rightarrow 0} K^{\prime} \lambda^{2} C^{2} \epsilon^{-2(\alpha / \beta+\eta)} \delta=0
$$

which proves weak $M_{1}$ convergence of $\left(A(T t)-\lambda \mu_{\zeta} T t\right) / b(T)$ to $X_{\alpha / \beta}(t)$.

Remark 4.2. If, instead of the $M$ function, we would have used the $J$-function, to attempt to prove $J_{1}$ convergence, the argument would be altered as follows. Since $\zeta$ is regularly varying with index $\beta, \zeta\left((1 / 2)^{1 / \beta} L_{k}\right) \sim(1 / 2) \zeta\left(L_{k}\right)$ for $L_{k}$ large. Heuristically, for each large job $\zeta\left(L_{k}\right)$, the halfway point $(1 / 2) \zeta\left(L_{k}\right)$ is also large. Take $t_{1}$ corresponding to the starting point of the large job, $t$ as halfway point, and $t_{2}$ corresponding to the endpoint of the large job, we see that if there is a 
large job arriving in the interval $\left[T t_{1}, T t_{2}\right]$, we can choose timepoints $T t_{1} \leq T t_{1}^{\prime} \leq T t^{\prime} \leq T t_{2}^{\prime} \leq T t_{2}$ such that $J\left(Z_{T}\left(t_{1}^{\prime}\right), Z_{T}\left(t_{2}^{\prime}\right), Z_{T}\left(t_{2}^{\prime}\right)\right)>\epsilon$. Therefore,

$$
\begin{aligned}
P\left(J\left(Z_{T}\left(t_{1}\right), Z_{T}(t), Z_{T}\left(t_{2}\right)\right)>\epsilon\right) & \cong P\left(\text { Large job arriving in }\left[T t_{1}, T t_{2}\right]\right) \\
& \cong P\left(\sum_{k=1}^{\left[\lambda T\left(t-t_{1}\right)\right]} \frac{\zeta\left(J_{k}^{(1)}\right)-E\left(\zeta\left(J_{1}^{(1)}\right)\right)}{b(T)}>\epsilon\right) \\
& +P\left(\sum_{k=1}^{\left[\lambda T\left(t_{2}-t\right)\right]} \frac{\zeta\left(J_{k}^{(2)}\right)-E\left(\zeta\left(J_{1}^{(2)}\right)\right)}{b(T)} \leq-\epsilon\right) \\
& \cong O\left(t_{2}-t_{1}\right)
\end{aligned}
$$

which is not enough to make

$P\left(J\left(Z_{T}\left(t_{1}\right), Z_{T}(t), Z_{T}\left(t_{2}\right)\right)>\epsilon\right)=O\left(\left(t_{2}-t_{1}\right)^{1+\beta}\right), \beta>0$.

\section{A SINGLE SERVER APPLICATION}

Suppose traffic modeled by (2.1) enters a network, and queues at an intermediary switch. Let the cumulative output at the switch by time $t$ be denoted by $R(t)$. Then the content of the fluid queue at time $T t$ is given by

$$
\begin{aligned}
Y(T t) & =A(T t)-R(T t)-\bigwedge_{s=0}^{T t}(A(s)-R(s)) \\
& =A(T t)-R(T t)-\bigwedge_{s=0}^{t}(A(T s)-R(T s)) .
\end{aligned}
$$

Furthermore, assume

$$
R(T t)=R_{T} t
$$

where

$$
\frac{R_{T}-\lambda \mu_{\zeta} T}{b(T)} \rightarrow c
$$

for some constant $c$. Then since the process $A(T \cdot)$ has no fixed discontinuities,

$$
\frac{A(T t)-R(T t)}{b(T)}=\frac{A(T t)-\lambda \mu_{\zeta} T t}{b(T)}-\left(\frac{R_{T}-\lambda \mu_{\zeta} T}{b(T)}\right) t \Rightarrow X_{\alpha / \beta}(t)-c t
$$

as $T \rightarrow \infty$ in $\left(D[0, \infty), M_{1}\right)$, where $X_{\alpha / \beta}(t)$ is the totally skewed Lévy motion described in Theorem 3.1. (See [30], Corollary 7.1).

The reflection mapping $x_{r}(t): x(t) \mapsto x(t)-\wedge_{s=0}^{t} x(s)$ is (Lipschitz) continuous in $\left(D[0, \infty), M_{1}\right)$ ([31], Theorem 3.2 and Remark 3.1; [30], Section 8). Therefore, we have by an application of the continuous mapping theorem:

$$
\frac{Y(T t)}{b(T)}=\frac{A(T t)-R(T t)-\bigwedge_{s=0}^{t}(A(T s)-R(T s))}{b(T)} \Rightarrow X_{\alpha / \beta}(t)-c t-\bigwedge_{s=0}^{t}\left(X_{\alpha / \beta}(s)-c s\right) .
$$

In particular, suppose $c=0$ in (5.1), which then becomes the 'heavy traffic' condition

$$
\frac{R_{T}-\lambda \mu_{\zeta} T}{b(T)} \rightarrow 0
$$


Then (5.2) yields

$$
\frac{Y(T t)}{b(T)} \Rightarrow X_{\alpha / \beta}(t)-\bigwedge_{s=0}^{t} X_{\alpha / \beta}(s) .
$$

as $T \rightarrow \infty$, in $\left(D[0, \infty), M_{1}\right)$. To illustrate the heavy traffic interpretation of this result, we argue as follows:

Consider the model of section 2 , but suppose we increase the arrival rate of source transmissions from $\lambda$ to $\lambda T$. Furthermore, we increase the speed of transmission. The new transmission schedule is given by $\zeta_{T}(t):=\zeta(T t)$, and the new transmission durations $L_{k}^{(T)}$ have the same distribution as $L_{k} / T$ :

$$
P\left(L_{k}^{(T)}>\frac{x}{T}\right)=P\left(L_{k}>x\right)=1-F(x)=x^{-\alpha} L(x)
$$

where $L(x)$ is as in (2.2). Then for the new system, the total traffic input by time $t, A_{T}(t)$, has the same distribution as $A(T t)$ above. Now consider a fluid queue with input $A_{T}(t)$, and output $R_{T}(t):=R_{T} t$. Denote its content at time $t$ by $Y_{T}(t)$. Under the heavy traffic condition (5.3), we see that in $\left(D[0, \infty), M_{1}\right)$ :

$$
\frac{Y_{T}(t)}{b(T)} \Rightarrow X_{\alpha / \beta}(t)-\bigwedge_{s=0}^{t} X_{\alpha / \beta}(s) \text { as } T \rightarrow \infty .
$$

\section{REFERENCES}

[1] A. Astrauskas. Limit theorems for sums of linearly generated random variables. Lithuanian Mathematical Journal, 23(2):127-134, 1983. Translated from Litovskii Matematicheskii Sbornik (Lieutuvos Matematikos Rinkinys).

[2] F. Avram and M. S. Taqqu. Probability bounds for M-Skorohod oscillations. Stochastic Processes and their Applications, 33:63-72, 1989.

[3] F. Avram and M. S. Taqqu. Weak convergence of sums of moving averages in the $\alpha$-stable domain of attraction. The Annals of Probability, 20:483-503, 1992.

[4] N.H. Bingham, C.M. Goldie, and J.L. Teugels. Regular Variation. Cambridge University Press, 1987.

[5] F. Brichet, J. Roberts, A. Simonian, and D. Veitch. Heavy traffic analysis of a storage model with long range dependent on/off sources. Queueing Systems, 23:197-215, 1996.

[6] Jason Cohen, Sidney Resnick, and Gennady Samorodnitsky. Sample correlations of infinite variance time series models: an empirical and theoretical study. J. Appl. Math. Stochastic Anal., 11(3):255-282, 1998.

[7] R.A. Davis and T. Mikosch. The sample acf of heavy tailed stationary processes with applications to arch. Preprint, 1997.

[8] L. de Haan. On Regular Variation and its Application to the Weak Convergence of Sample Extremes. Mathematisch Centrum Amsterdam, 1970.

[9] P. Feigin and S. Resnick. Pitfalls of fitting autoregressive models for heavy-tailed time series. Available at http://www.orie.cornell.edu/trlist/trlist.html at TR1163.ps.Z; to appear: Extremes., 1999.

[10] W. Feller. An Introduction to Probability Theory and its Applications, volume 2. Wiley, New York, 2nd edition, 1971.

[11] M.W. Garrett and W. Willinger. Analysis, modeling and generation of self similar vbr video traffic. In $A C M$ SigComm, London, 1994.

[12] J. L. Geluk and L. de Haan. Regular Variation, Extensions and Tauberian Theorems, volume 40 of CWI Tract. Stichting Mathematisch Centrum, Centrum voor Wiskunde en Informatica, Amsterdam, 1987.

[13] D. Heath, S. Resnick, and G. Samorodnitsky. Patterns of buffer overflow in a class of queues with long memory in the input stream. Ann. Appl. Probab., 7(4):1021-1057, 1997.

[14] D. Heath, S. Resnick, and G. Samorodnitsky. Heavy tails and long range dependence in on/off processes and associated fluid models. Math. Oper. Res., 23(1):145-165, 1998.

[15] P.R. Jelenković and A.A. Lazar. Asymptotic results for multiplexing subexponential on-off processes. Advances in Applied Probability, 31, 1999. To appear.

[16] Predrag R. Jelenković and Aurel A. Lazar. A network multiplexer with multiple time scale and subexponential arrivals. In Stochastic Networks (New York, 1995), pages 215-235. Springer, New York, 1996. 
[17] Takis Konstantopoulos and Si-Jian Lin. Macroscopic models for long-range dependent network traffic. Queueing Systems Theory Appl., 28(1-3):215-243, 1998.

[18] W.E. Leland, M.S. Taqqu, W. Willinger, and D.V. Wilson. On the self-similar nature of Ethernet traffic (extended version). IEEE/ACM Transactions on Networking, 2:1-15, 1994.

[19] M. Parulekar and A. M. Makowski. Tail probabilities for a multiplexer with a self-similar traffic. Proceedings of the 15th Annual IEEE INFOCOM, pages 1452-1459, 1996.

[20] S. Resnick. Heavy tail modeling and teletraffic data. Ann. Statist., 25:1805-1869, 1997.

[21] S. Resnick. Why non-linearities can ruin the heavy tailed modeler's day. In Murad S. Taqq Robert Adler, Raisa Feldman, editor, A PRACTICAL GUIDE TO HEAVY TAILS: Statistical Techniques for Analysing Heavy Tailed Distributions. Birkhäuser, Boston, 1998.

[22] S. Resnick and G. Samorodnitsky. A heavy traffic limit theorem for workload processes with heavy tailed service requirements. Technical report 1221, available at www.orie.cornell.edu/trlist/trlist.html; to appear: Management Science, 1998.

[23] S. Resnick, G. Samorodnitsky, and F. Xue. Growth rates of sample covariances of stationary symmetric $\alpha$-stable processes associated with null recurrent markov chains. Technical report TR1220; available at www.orie.cornell.edu/trlist/trlist.html, 1998.

[24] S. Resnick, G. Samorodnitsky, and F. Xue. How misleading can sample acf's of stable ma's be? (very!). Technical report 1209; available at www.orie.cornell.edu/trlist/trlist.html; to appear: Annals of Applied Probability, 1998.

[25] S. Resnick and E. van den Berg. A test for nonlinearity of time series with infinite variance. Technical report 1222, available at www.orie.cornell.edu/trlist/trlist.html, 1998.

[26] S.I. Resnick. Extreme Values, Regular Variation and Point Processes. Springer-Verlag, New York, 1987.

[27] A.V. Skorohod. Limit theorems for stochastic processes. Theory of Probability and Its Apllications, 1(3):261-290, 1956.

[28] M. Taqqu, W. Willinger, and R. Sherman. Proof of a fundamental result in self-similar traffic modeling. Computer Communications Review, 27:5-23, 1997.

[29] Ward Whitt. Limits for cumulative input processes to queues. Preprint, AT\&T Labs, 1999.

[30] Ward Whitt. On the Skorohod $M$-topologies. Preprint, AT\&T Labs, 1999.

[31] Ward Whitt. The reflection map is Lipschitz with appropriate Skorohod $M$-metrics. Preprint, AT\&T Labs, 1999.

[32] W. Willinger and V. Paxson. Where mathematics meets the internet. Notices of the American Mathematical Society, 45(8):961-970, 1998.

[33] W. Willinger, M.S. Taqqu, M. Leland, and D. Wilson. Self-similarity in high-speed packet traffic: analysis and modelling of ethernet traffic measurements. Statistical Science, 10:67-85, 1995.

[34] W. Willinger, M.S. Taqqu, M. Leland, and D. Wilson. Self-similarity through high variability: statistical analysis of ethernet lan traffic at the source level. Computer Communications Review, 25:100-113, 1995. Proceedings of the ACM/SIGCOMM'95, Cambridge, MA.

[35] W. Willinger, M.S. Taqqu, M. Leland, and D. Wilson. Self-similarity through high variability: statistical analysis of ethernet lan traffic at the source level (extended version). IEEE/ACM Transactions on Networking, 1996. To appear.

School of Operations Research and Industrial Engineering, Cornell University, IthaCA, Ny 14853 E-mail address: sid@orie.cornell.edu

Center for Applied Mathematics, Cornell University, Ithaca, NY 14853

E-mail address: ericvdb@cam.cornell.edu 